\title{
Reinforced walk on graphs and neural networks
}

\author{
by \\ Józef MYjAK (Aquila and Kraków) and RyszARD Rudnicki (Katowice) \\ Dedicated to Professor Jan Kisyński on the occasion of his 75th birthday
}

\begin{abstract}
A directed-edge-reinforced random walk on graphs is considered. Criteria for the walk to end up in a limit cycle are given. Asymptotic stability of some neural networks is shown.
\end{abstract}

1. Introduction. There are a large number of different variants of random walk with elements of memory [19]. Let us mention only self-attracting diffusion [2], [18], self-avoiding walk [14], self-interacting random walk [22], reinforced random walk, etc. A reinforced random walk, which is the main subject of our paper, is defined on the lattice $\mathbb{Z}^{d}$ or on graphs and the general rule is that it prefers earliest visited paths. One can consider vertex or edge-reinforced random walks. A vertex-reinforced random walk was introduced by Coppersmith and Diaconis [3] and it has been studied later by Pemantle [17], Davis [4], Sellke [21], Benaïm [1], Volkov [23], Davis and Volkov [5] and others. Edge-reinforced random walks were investigated by Pemantle [16], Lyons and Pemantle [13], Mauldin, Monticino, and von Weizsäcker [15], Horváth and Shao [8], Keane and Rolles [10], [11], Rudnicki and Wolf [20], Limic [12] and others.

In this paper we study a new class of edge-reinforced random walks on graphs. At each time, each vertex $a$ has an associated vector $\left\{p_{a b}\right\}_{b \in \mathcal{E}(a)}$ containing the probabilities for jumping from $a$ to each neighbour of $b$. Here $\mathcal{E}(a)$ denotes the set of all ends of edges which begin at $a$. When the walk-

2000 Mathematics Subject Classification: Primary 60K37; Secondary 60J10, 60J20, 60G50, 82C41.

Key words and phrases: reinforced random walk on graphs, limit cycle, neural network.

This research was partially supported by the State Committee for Scientific Research (Poland) Grant No. N N201 021133 and by EC FP6 Marie Curie ToK programme SPADE2, MTKD-CT-2004-014508 and Polish MNiSW SPB-M. This work was written while R.R. was a visitor to the University of L'Aquila. The authors thank the group GNAMPA (CNR) for financial support of that visit. 
ing point jumps from $a$ to a neighbouring vertex $b$, the probability vector $\left\{p_{a b}\right\}_{b \in \mathcal{E}(a)}$ is replaced by a new one. We show, under certain conditions, that the walk ends up in a limit cycle with probability one. It should be mentioned that our walk is not Markovian because the probability of choosing any direction depends on the past. If we extend the phase space by adding the distributions of the probabilities of passing particular edges we obtain a Markov process.

The main role in our model is played by the learning process [9] which describes how the vector $\left\{p_{a b}\right\}_{b \in \mathcal{E}(a)}$ is changed. This process is defined in Section 2 and it is called a random decision system. The probability of choosing a given edge depends on the state of the system and the changes of the states after making some decision (i.e. the choice of an edge) are defined by a transition probability function. The main result of that section is that under certain assumptions the system eventually takes the same decision.

In Section 3 we study edge-reinforced random walks on graphs. We have independent decision systems at each vertex and these decision systems are responsible for the choice of the next vertex. We consider both finite and infinite graphs. The stabilization of decision systems at vertices which were visited many times causes that the random walker will go along a limit cycle. Our theorems generalize those from [20], where the edge-reinforced random walk was considered on the lattice $\mathbb{Z}^{d}$ and the decision system was defined in the same way at each point of $\mathbb{Z}^{d}$ by means of an iterated function system.

The last section is devoted to artificial neural networks. We consider a simple model of a neural network which is an oriented graph with input and output vertices. Vertices and oriented edges play the role of, respectively, neurons and synaptic connections. The neural network is learning using a reinforcement procedure similar to the previously described one for the random walk on graphs. At each vertex except the output vertices we have a decision system. We begin the process of learning at a randomly chosen input vertex. Then we go through the network choosing edges according to the rules given by the decision systems. When we reach an output vertex we repeat the procedure, etc. This kind of learning system is based on hebbian synaptic modification rules [6], [7]. After some time the network "stabilizes", i.e. a path from a given input vertex is strictly determined and if two paths meet at some vertex then they further coincide. This means that with high probability similar inputs (i.e. starting from near vertices) produce the same output.

2. Random decision system. As mentioned in the introduction, the probabilities of jumping from a vertex to its neighbour change in time and each vertex has its own random decision system (independent of other verticies) which determines these probabilities. We start with an informal def- 
inition of a decision system. At each time step a system takes a decision $i$. The probability of taking this decision depends on the state $x$ of the system and equals $p_{i}(x)$. But if we take a decision $i$ then the state of the system will change to a new state $y$ determined by some transition probability function $P_{i}(x, \cdot)$. Now we proceed to a formal definition.

Let $X$ be a measurable space with a $\sigma$-algebra $\mathcal{A}$. Let $D$ be a nonempty countable set. Assume that for each $i \in D$ we have transition probability functions $P_{i}: X \times \mathcal{A} \rightarrow[0,1]$, that is:

(a) for each $i \in D$ and $x \in X$ the function $A \mapsto P_{i}(x, A)$ is a probability measure,

(b) for each $i \in D$ and $A \in \mathcal{A}$ the function $x \mapsto P_{i}(x, A)$ is measurable.

Let $p_{i}: X \rightarrow[0,1], i \in D$, be measurable functions such that

$$
\sum_{i \in D} p_{i}(x)=1 \quad \text { for } x \in X
$$

The system $\left(X, \mathcal{A},\left\{P_{i}\right\}_{i \in D},\left\{p_{i}\right\}_{i \in D}\right)$ will be called a random decision system. The sets $X, D$ and $\widetilde{X}=X \times D$ are called, respectively, the state space, the set of decisions, and the decision space. Having a random decision system we can introduce two Markov processes (or Markov families).

Let $\widetilde{\mathcal{A}}$ be the product $\sigma$-algebra $\mathcal{A} \times 2^{D}$. Then for each $x \in X, i \in D$, $A \in \mathcal{A}$ and $I \subset D$ we put

$$
\widetilde{P}((x, i), A \times I)=\sum_{j \in I} p_{j}(x) P_{j}(x, A) .
$$

We can extend the function $\widetilde{P}$ onto the set $\widetilde{X} \times \widetilde{\mathcal{A}}$ in such a way that $\widetilde{P}$ is a transition probability function. Then there exists a homogeneous Markov process $\left(\widetilde{\xi}_{n}\right)_{n=0}^{\infty}$ corresponding to $\widetilde{P}$. This means that we have some probability space $\left(\Omega, \Sigma\right.$, Prob) and a sequence $\left(\widetilde{\xi}_{n}\right)_{n=0}^{\infty}$ of random elements $\widetilde{\xi}_{n}: \Omega \rightarrow \widetilde{X}$ such that the sequence $\widetilde{\xi}_{n}$ is a Markov process and

$$
\operatorname{Prob}\left(\widetilde{\xi}_{n+1} \in \widetilde{A} \mid \widetilde{\xi}_{n}=\widetilde{x}\right)=\widetilde{P}(\widetilde{x}, \widetilde{A}) .
$$

Let $\widetilde{\xi}_{n}(\omega)=\left(\xi_{n}(\omega), k_{n}(\omega)\right)$ for $n=0,1, \ldots$ Then $\left(\xi_{n}\right)_{n=0}^{\infty}$ is a homogeneous Markov process. The process $\left(\xi_{n}\right)_{n=0}^{\infty}$ has the transition probability function

$$
P(x, A)=\sum_{i \in D} p_{i}(x) P_{i}(x, A) \quad \text { for } x \in X, A \in \mathcal{A},
$$

that is,

$$
\operatorname{Prob}\left(\xi_{n+1} \in A \mid \xi_{n}=x\right)=P(x, A) .
$$

The random elements $\xi_{n}, k_{n}$ describe, respectively, the state of the random decision system at time $t=n$ and the decision taken at time $t=n-1\left(k_{0}\right.$ 
can be arbitrary). We have

$$
\begin{aligned}
\operatorname{Prob}\left(k_{n+1}=k \mid \xi_{n}=x\right) & =p_{k}(x), \\
\operatorname{Prob}\left(\xi_{n+1} \in A \mid \xi_{n}=x, k_{n+1}=k\right) & =P_{k}(x, A),
\end{aligned}
$$

for $x \in X$ and $A \in \mathcal{A}$.

The sequence $\left(k_{n}\right)$ of random variables is called a decision process. A trajectory $\left(k_{n}(\omega)\right)$ is called eventually constant if there are $k(\omega) \in D$ and $n_{0}(\omega) \in \mathbb{N}$ such that $k_{n}(\omega)=k(\omega)$ for $n \geq n_{0}(\omega)$. A random decision system $\left(X, \mathcal{A},\left\{P_{i}\right\}_{i \in D},\left\{p_{i}\right\}_{i \in D}\right)$ is called eventually constant if almost all trajectories are eventually constant.

Now, we give an example of a random decision system.

EXAmple 1 (An iterated function system). Let $T_{i}: X \rightarrow X, i \in D$, be measurable transformations and $p_{i}: X \rightarrow[0,1], i \in D$, be measurable functions which satisfy (1). If we put $P_{i}(x, A)=\mathbf{1}_{A}\left(T_{i}(x)\right)$ for $x \in X$ and $A \in \mathcal{A}$, then $P_{i}$ is a transition probability function. The system $\left(X, \mathcal{A},\left\{T_{i}\right\}_{i \in D},\left\{p_{i}\right\}_{i \in D}\right)$ will be called an iterated function system. In this case we have $\xi_{n+1}=T_{k_{n+1}}\left(\xi_{n}\right)$.

Let $\left(X, \mathcal{A},\left\{P_{i}\right\}_{i \in D},\left\{p_{i}\right\}_{i \in D}\right)$ be a random decision system. For $x \in X$ and $A \in \mathcal{A}$ we set $Q_{i}(x, A)=p_{i}(x) P_{i}(x, A), Q_{i}^{1}(x, A)=Q_{i}(x, A)$ and

$$
Q_{i}^{n+1}(x, A)=\int_{X} Q_{i}(y, A) Q_{i}^{n}(x, d y) .
$$

From the definition of $Q_{i}^{n}$ it follows immediately that

$$
Q_{i}^{1}(x, X) \geq Q_{i}^{2}(x, X) \geq \cdots \geq 0 \quad \text { for each } x \in X .
$$

The main result of this section is the following

Theorem 1. Let $\left(X, \mathcal{A},\left\{P_{i}\right\}_{i \in D},\left\{p_{i}\right\}_{i \in D}\right)$ be a random decision system and let $c>0$ be a constant. Assume that for each $x \in X$ there is an $i \in D$ such that

$$
Q_{i}^{n}(x, X)>c \quad \text { for } n \geq 1 \text {. }
$$

Then the random decision system $\left(X, \mathcal{A},\left\{P_{i}\right\}_{i \in D},\left\{p_{i}\right\}_{i \in D}\right)$ is eventually constant.

Corollary 1. Let $\left(X, \mathcal{A},\left\{T_{i}\right\}_{i \in D},\left\{p_{i}\right\}_{i \in D}\right)$ be an iterated function system and let $c>0$ be a constant. Assume that for each $x \in X$ there is an $i \in D$ such that

$$
\prod_{n=0}^{\infty} p_{i}\left(T_{i}^{n}(x)\right)>c .
$$

Then the random decision system $\left(X, \mathcal{A},\left\{P_{i}\right\}_{i \in D},\left\{p_{i}\right\}_{i \in D}\right)$ is eventually constant. 
REMARK 1. Condition (9) cannot be replaced by a weaker one: for each $x \in X$ there is $i \in D$ such that

$$
\prod_{n=0}^{\infty} p_{i}\left(T_{i}^{n}(x)\right)>0 .
$$

Indeed, let $X=\mathbb{Z}, D=\{1,2\}, T_{1}(x)=x+1, T_{2}(x)=x-1, p_{1}(x)=1-x^{-2}$ for $x \geq 2$ and $p_{1}(x)=1 / 3$ for $x \leq 1$. Then

$$
\prod_{n=0}^{\infty} p_{1}\left(T_{1}^{n}(x)\right)>0 \quad \text { for each } x \in X
$$

and

$$
\operatorname{Prob}\left(\lim _{n \rightarrow \infty} \xi_{n}=-\infty \mid \xi_{0}=x\right)>0 \quad \text { for each } x \in X .
$$

This means that there exists a subset $\Omega_{1}$ of $\Omega$ with $\operatorname{Prob}\left(\Omega_{1}\right)>0$ such that for each $\omega \in \Omega_{1}$ we have $k_{n}(\omega)=2$ for an infinite number of $n$ 's. On the other hand, the probability that $k_{n}=2$ for all but finitely many $n$ equals zero.

REMARK 2. Inequality (9) can be written in the following way:

$$
\prod_{n=0}^{\infty} \int_{X} p_{i}(y) P_{i}^{n}(x, d y)>c,
$$

where $P_{i}^{1}(x, A)=P_{i}(x, A)$ and $P_{i}^{n+1}(x, A)=\int_{X} P_{i}(y, A) P_{i}^{n}(x, d y)$. It is interesting that condition (8) of Theorem 1 cannot be replaced by (11). Consider a random decision system $\left(X, \mathcal{A}, P_{1}, P_{2}, p_{1}, p_{2}\right)$ on the space $X=$ $\{1,2,3,4\}, \mathcal{A}=2^{X}$. Let $p_{i j}^{n}=P_{1}^{n}(i,\{j\}), q_{i j}^{n}=P_{2}^{n}(i,\{j\}), p_{i j}=p_{i j}^{1}, q_{i j}=$ $q_{i j}^{1}$ and assume that

$$
\begin{gathered}
p_{1}(1)=p_{1}(3)=0, \quad p_{1}(2)=p_{1}(4)=1, \\
p_{11}=1, \quad p_{22}=p_{23}=\frac{1}{2}, \quad p_{34}=1, \quad p_{44}=1, \\
q_{11}=1, \quad q_{21}=1, \quad q_{32}=q_{33}=\frac{1}{2}, \quad q_{44}=1 .
\end{gathered}
$$

Since $p_{22}^{n}=2^{-n}$ and $p_{24}^{n}=1-2^{1-n}$ for $n \geq 1$, we have

$$
\int_{X} p_{1}(y) P_{1}^{n}(2, d y)=p_{22}^{n}+p_{24}^{n}=1-2^{-n}
$$

and since $p_{22}^{0}=1$ we obtain

$$
\prod_{n=0}^{\infty} \int_{X} p_{1}(y) P_{1}^{n}(2, d y)=c,
$$

where $c=\prod_{n=1}^{\infty}\left(1-2^{-n}\right)$. Since $p_{44}^{n}=1$ for all $n \geq 0$, inequality (11) holds for $x=4$ and $i=1$. Analogously, we can check that (11) holds for $x=1,3$ 
and $i=2$. On the other hand,

$$
\begin{aligned}
& \operatorname{Prob}\left(\xi_{n+1}=2 \mid \xi_{n}=2\right)=\operatorname{Prob}\left(\xi_{n+1}=3 \mid \xi_{n}=2\right)=\frac{1}{2}, \\
& \operatorname{Prob}\left(\xi_{n+1}=2 \mid \xi_{n}=3\right)=\operatorname{Prob}\left(\xi_{n+1}=3 \mid \xi_{n}=3\right)=\frac{1}{2},
\end{aligned}
$$

which implies that if $\xi_{0}=2$ then the process $\xi_{n}$ takes each of the values 2 and 3 for infinitely many $n$.

We precede the proof of Theorem 1 by some definitions and a lemma. Let

$$
\varphi_{i}(x)=\lim _{n \rightarrow \infty} Q_{i}^{n}(x, X)
$$

We define

$$
X_{i, \varepsilon}=\left\{x \in X: \varphi_{i}(x) \geq 1-\varepsilon\right\}, \quad X_{\varepsilon}=\bigcup_{i \in D} X_{i, \varepsilon},
$$

and let $P^{n}(x, A)$ be the $n$-step transition probability function for the process $\xi_{n}$.

Lemma 1. Assume that (8) holds. Then for every $x \in X$ and $\varepsilon>0$ we have

$$
\lim _{n \rightarrow \infty} P^{n}\left(x, X_{\varepsilon}\right)=1 .
$$

Proof. Fix $x \in X$ and $\varepsilon>0$. Let $i \in D$ be a decision such that $\varphi_{i}(x) \geq c$. From (6) and (12) it follows that

$$
\varphi_{i}(x)=\int_{X} \varphi_{i}(y) Q_{i}^{n}(x, d y) .
$$

Let

$$
\mu_{n}(A)=\frac{Q_{i}^{n}(x, A)}{Q_{i}^{n}(x, X)} .
$$

Then $\mu_{n}$ is a probability measure. From the definition of $\varphi_{i}$ it follows that there exists an integer $n_{0}(x)$ such that

$$
\frac{\varphi_{i}(x)}{Q_{i}^{n}(x, X)} \geq 1-\varepsilon^{2} \quad \text { for } n \geq n_{0}(x) .
$$

From (13)-(15) we obtain

$$
\int_{X} \varphi_{i}(y) \mu_{n}(d y) \geq 1-\varepsilon^{2}
$$

and consequently

$$
\int_{X}\left(1-\varphi_{i}(y)\right) \mu_{n}(d y) \leq \varepsilon^{2} .
$$


Since $\varphi_{i} \leq 1$, the Chebyshev inequality and (16) imply

$$
\mu_{n}\left(y \in X: 1-\varphi_{i}(y) \geq \varepsilon\right) \leq \frac{1}{\varepsilon} \int_{X}\left(1-\varphi_{i}(y)\right) \mu_{n}(d y) \leq \varepsilon .
$$

This means that

$$
\mu_{n}\left(X_{i, \varepsilon}\right) \geq 1-\varepsilon
$$

From the definition of $\mu_{n}$ it follows that

$$
Q_{i}^{n}\left(x, X_{i, \varepsilon}\right) \geq(1-\varepsilon) Q_{i}^{n}(x, X) \geq(1-\varepsilon) \varphi_{i}(x) .
$$

Since

$$
P^{n}\left(x, X_{\varepsilon}\right) \geq P^{n}\left(x, X_{i, \varepsilon}\right) \geq Q_{i}^{n}\left(x, X_{i, \varepsilon}\right) \geq(1-\varepsilon) \varphi_{i}(x)
$$

for $n \geq n_{0}(x)$, we obtain

$$
\begin{array}{ll}
\liminf _{n \rightarrow \infty} P^{n}\left(y, X_{\varepsilon}\right) \geq(1-\varepsilon) c & \text { for } y \in X, \\
\liminf _{n \rightarrow \infty} P^{n}\left(y, X_{\varepsilon}\right) \geq(1-\varepsilon)^{2} & \text { for } y \in X_{\varepsilon} .
\end{array}
$$

From the Fatou lemma and the formula

$$
P^{n+m}\left(x, X_{\varepsilon}\right)=\int_{X_{\varepsilon}} P^{n}\left(y, X_{\varepsilon}\right) P^{m}(x, d y)+\int_{X \backslash X_{\varepsilon}} P^{n}\left(y, X_{\varepsilon}\right) P^{m}(x, d y)
$$

we have

$$
\begin{aligned}
\liminf _{n \rightarrow \infty} P^{n+m}\left(x, X_{\varepsilon}\right) & \geq(1-\varepsilon)^{2} P^{m}\left(x, X_{\varepsilon}\right)+(1-\varepsilon) c P^{m}\left(x, X \backslash X_{\varepsilon}\right) \\
& =c(1-\varepsilon)+(1-\varepsilon-c)(1-\varepsilon) P^{m}\left(x, X_{\varepsilon}\right) .
\end{aligned}
$$

Fix $x \in X$ and let $\alpha(\varepsilon)=\liminf _{n \rightarrow \infty} P^{n}\left(x, X_{\varepsilon}\right)$. Then from the last inequality we obtain

$$
\alpha(\varepsilon) \geq c(1-\varepsilon)+(1-\varepsilon-c)(1-\varepsilon) \alpha(\varepsilon)
$$

and consequently

$$
\alpha(\varepsilon) \geq \frac{c(1-\varepsilon)}{1-(1-\varepsilon-c)(1-\varepsilon)} .
$$

Since $\alpha$ is a nondecreasing function and $\alpha(\varepsilon) \leq 1$ inequality (20) implies

$$
1 \geq \alpha(\varepsilon) \geq \lim _{\varepsilon \rightarrow 0} \alpha(\varepsilon) \geq 1
$$

and $\alpha(\varepsilon)=1$ for each $\varepsilon>0$, which completes the proof.

Proof of Theorem 1. Let $X^{k}=X \times\{k\}$ for $k \in D$. For every $(x, i) \in \widetilde{X}$ and $A \subset X$ we have

$$
\widetilde{P}((x, i), A \times\{k\})=p_{k}(x) P_{k}(x, A)=Q_{k}(x, A) .
$$


Since $\widetilde{P}$ is the probability transition function of the process $\widetilde{\xi}_{n}$, we have

$$
\begin{aligned}
\operatorname{Prob} & \left(k_{n+1}=k_{n+2}=\cdots=k_{n+m}=k \mid \widetilde{\xi}_{n}=(x, i)\right) \\
& =\operatorname{Prob}\left(\widetilde{\xi}_{n+1} \in X^{k}, \ldots, \widetilde{\xi}_{n+m} \in X^{k} \mid \widetilde{\xi}_{n}=(x, i)\right) \\
& =\int_{X^{k}} \ldots \int_{X^{k}} \widetilde{P}\left((x, i), d \widetilde{y}_{1}\right) \widetilde{P}\left(\widetilde{y}_{1}, d \widetilde{y}_{2}\right) \ldots \widetilde{P}\left(\widetilde{y}_{m-1}, d \widetilde{y}_{m}\right) \\
& =\int_{X} \ldots \int_{X} Q_{k}\left(x, d y_{1}\right) Q_{k}\left(y_{1}, d y_{2}\right) \ldots Q_{k}\left(y_{m-1}, d y_{m}\right)=Q_{k}^{m}(x, X) .
\end{aligned}
$$

This implies that

$$
\operatorname{Prob}\left(k_{n+1}=k_{n+2}=\cdots=k_{n+m}=k \mid \xi_{n}=x\right)=Q_{k}^{m}(x, X)
$$

and consequently

$$
\operatorname{Prob}\left(k_{m}=k \text { for all } m>n \mid \xi_{n}=x\right)=\varphi_{k}(x) .
$$

Let $\varepsilon \in(0,1 / 2)$ be a given number. Since

$$
\operatorname{Prob}\left(\xi_{n} \in X_{\varepsilon}\right)=\int_{\Omega} P^{n}\left(\xi_{0}(\omega), X_{\varepsilon}\right) \operatorname{Prob}(d \omega),
$$

from Lemma 1 and the Lebesgue theorem it follows that

$$
\lim _{n \rightarrow \infty} \operatorname{Prob}\left(\xi_{n} \in X_{\varepsilon}\right)=1 .
$$

Let $n_{0}$ be a positive integer such that

$$
\operatorname{Prob}\left(\xi_{n_{0}} \in X_{\varepsilon}\right) \geq 1-\varepsilon .
$$

Let $A=\left\{\omega: \xi_{n_{0}} \in X_{\varepsilon}\right\}$ and $A_{k}=\left\{\omega: \xi_{n_{0}} \in X_{k, \varepsilon}\right\}$ for $k \in D$. Then $A=\bigcup_{k \in D} A_{k}$. Let

$$
B_{k}=\left\{\omega: k_{n}=k \text { for } n \geq n_{0}\right\}, \quad B=\bigcup_{k \in D} B_{k} .
$$

Then from (21) we obtain

$$
\operatorname{Prob}\left(B_{k} \mid \xi_{n_{0}}=x\right)=\varphi_{k}(x) .
$$

Since $\varphi_{k}(x) \geq 1-\varepsilon$ for $x \in X_{k, \varepsilon}$ we have

$$
\operatorname{Prob}\left(B_{k} \mid A_{k}\right) \geq 1-\varepsilon \text {. }
$$

Consequently,

$$
\operatorname{Prob}\left(B_{k}\right) \geq \operatorname{Prob}\left(B_{k} \cap A_{k}\right) \geq(1-\varepsilon) \operatorname{Prob}\left(A_{k}\right) .
$$

Since $B_{1}, \ldots, B_{N}$ are pairwise disjoint we obtain

$$
\operatorname{Prob}(B) \geq(1-\varepsilon) \operatorname{Prob}(A) \geq(1-\varepsilon)^{2} .
$$

Since $\varepsilon$ is any positive constant, inequality (24) implies that for almost every $\omega$ there is $k(\omega)$ such that $k_{n}(\omega)=k(\omega)$ for sufficiently large $n$, which completes the proof. 
ExAmple 2. Now we give an example of an iterated function system which satisfies the assumptions of our theorem. Let

$$
X=\left\{x \in[0,1]^{N}: x_{1}+\cdots+x_{N}=1\right\}, \quad p_{i}(x)=x_{i} \quad \text { for } x \in X,
$$

and we assume that the transformations $T_{i}: X \rightarrow X, i=1, \ldots, N$, satisfy the inequality

$$
1-\left(T_{i}(x)\right)_{i} \leq \varphi\left(1-x_{i}\right),
$$

where $\left(T_{i}(x)\right)_{i}$ is the $i$ th coordinate of $T_{i}(x)$. We assume that $\varphi:[0,1] \rightarrow$ $[0,1]$ is an increasing function such that

$$
\sum_{k=1}^{\infty} \varphi^{k}(1-1 / N)<\infty
$$

where $\varphi^{k}(t)=\varphi \circ \varphi^{k-1}(t)$ for $k \geq 1$. Since $\varphi$ is an increasing function, inequality (25) implies

$$
1-\left(T_{i}^{n}(x)\right)_{i} \leq \varphi^{n}\left(1-x_{i}\right)
$$

From (26) it follows that

$$
p_{i}\left(T_{i}^{n}(x)\right) \geq 1-\varphi^{n}\left(1-x_{i}\right) .
$$

Since $x_{i} \geq 1 / N$ for some $i$, inequalities (26) and (28) imply

$$
\prod_{n=0}^{\infty} p_{i}\left(T_{i}^{n}(x)\right) \geq \prod_{n=0}^{\infty}\left(1-\varphi^{n}\left(1-x_{i}\right)\right) \geq c>0
$$

where $c=\prod_{n=0}^{\infty}\left(1-\varphi^{n}(1-1 / N)\right)$.

If for example there exists $\lambda<1$ such that $\left(T_{i}(x)\right)_{j} \leq \lambda x_{j}$ for $x_{i} \geq 1 / N$ and all $j \neq i$, then (25) and (26) hold with $\varphi(t)=\lambda t$ for $t \in[0,1-1 / N]$.

3. Reinforced random walk on graphs. Let $\mathcal{Z}$ be a nonempty set and let $\mathcal{K}$ be a nonempty subset of $\mathcal{Z} \times \mathcal{Z}$. A pair $(\mathcal{Z}, \mathcal{K})$ is a directed graph, $\mathcal{Z}$ is the set of its vertices and $\mathcal{K}$ is the set of its oriented edges. If $(a, b) \in \mathcal{K}$ then $a$ and $b$ are called, respectively, the beginning and end of the edge $(a, b)$. For any vertex $a \in \mathcal{Z}$ we set

$$
\mathcal{E}(a)=\{b \in \mathcal{Z}:(a, b) \in \mathcal{K}\}, \quad \mathcal{B}(a)=\{b \in \mathcal{Z}:(b, a) \in \mathcal{K}\} .
$$

Now we assume that the set $\mathcal{Z}$ is finite and that for each vertex $a$ the set $\mathcal{E}(a)$ is nonempty. Moreover, we assume that at each vertex $a$ we have a random decision system

$$
\mathcal{S}_{a}=\left(X, \mathcal{A},\left\{P_{a b}\right\}_{b \in \mathcal{E}(a)},\left\{p_{a b}\right\}_{b \in \mathcal{E}(a)}\right) .
$$

For simplicity we assume that the set $X$ and the $\sigma$-algebra $\mathcal{A}$ are the same for all vertices.

Now we describe a random walk on the graph $(\mathcal{Z}, \mathcal{K})$. At the beginning all random decision systems are in state $x_{0} \in X$. We begin the walk at some 
vertex $a_{0}$. We choose an adjacent vertex $b_{i} \in \mathcal{E}\left(a_{0}\right)$ with the probability $p_{a_{0} b_{i}}\left(x_{0}\right)$ and we go to the vertex $b_{i}$. We also change the state of the random decision system $\mathcal{S}_{a_{0}}$. The distribution of the new state of $\mathcal{S}_{a_{0}}$ is given by the probability measure $\mu(A)=P_{a_{0} b_{i}}\left(x_{0}, A\right)$. At the next vertex, say $b$, we choose an adjacent vertex and change the state of $\mathcal{S}_{b}$ according to the same law as at $a_{0}$, and so on.

The state of the random walk at any time $t$ is described by the position of the moving point and the states of all random decision systems $\mathcal{S}_{a}, a \in \mathcal{Z}$.

We denote by $\eta_{n}$ the position of the moving point and by $\chi_{n}^{a}$ the state of $\mathcal{S}_{a}$ at time $t=n$. Set $\chi_{n}=\left(\chi_{n}^{a}\right)_{a \in \mathcal{Z}}$. Then $\left(\eta_{n}, \chi_{n}\right)$ is a Markov process on some complete probability space $\left(\Omega, \Sigma\right.$, Prob) with values in $\mathcal{Z} \times X^{\mathcal{Z}}$ satisfying the following conditions:

(a) $\eta_{0}=a_{0}, \chi_{0}^{a}=x_{0}$ for every $a \in \mathcal{Z}$,

(b) for all $x \in X, A \in \mathcal{A}, a \in \mathcal{Z}, b \in \mathcal{E}(a)$ we have

$$
\operatorname{Prob}\left(\eta_{n+1}=b, \chi_{n+1}^{a} \in A \mid \eta_{n}=a, \chi_{n}^{a}=x\right)=p_{a b}(x) P_{a b}(x, A),
$$

(c) for all $x \in X, a, b \in \mathcal{Z}, a \neq b$, we have

$$
\operatorname{Prob}\left(\chi_{n+1}^{b}=x \mid \eta_{n}=a, \chi_{n}^{b}=x\right)=1 .
$$

A sequence $\left(a_{1}, \ldots, a_{m}\right)$ of different vertices is called a cycle if $a_{i+1} \in$ $\mathcal{E}\left(a_{i}\right)$ for $i \in\{1, \ldots, m-1\}$ and $a_{1} \in \mathcal{E}\left(a_{m}\right)$. We say that a sequence $\left(b_{n}\right)_{n \in \mathbb{N}}$ of vertices has a limit cycle if there is a cycle $\left(a_{1}, \ldots, a_{m}\right)$ and a positive integer $n_{0}$ such that $b_{n_{0}+i+k m}=a_{i}$ for $i \in\{1, \ldots, m\}$ and $k=0,1, \ldots$

As in the previous section we introduce functions

$$
\begin{aligned}
Q_{a b}(x, A) & =p_{a b}(x) P_{a b}(x, A), \quad Q_{a b}^{n+1}(x, A)=\int_{X} Q_{a b}(y, A) Q_{a b}^{n}(x, d y), \\
\varphi_{a b}(x) & =\lim _{n \rightarrow \infty} Q_{a b}^{n}(x, X) .
\end{aligned}
$$

Now we can formulate the following theorem.

TheOREM 2. Consider a random walk on a graph $(\mathcal{Z}, \mathcal{K})$ and let $c>0$ be a given number. Assume that for each $a \in \mathcal{Z}$ and $x \in X$ there is $b \in \mathcal{E}(a)$ such that $\varphi_{a b}(x) \geq c$. Then there exists a measurable subset $\Omega_{0} \subset \Omega$ such that $\operatorname{Prob}\left(\Omega_{0}\right)=1$ and for each $\omega \in \Omega_{0}$ the sequence $\left\{\xi_{n}(\omega)\right\}_{n=0}^{\infty}$ has a limit cycle.

Proof. We denote by $A_{a}^{1}$ the subset of $\Omega$ which consists of all $\omega \in \Omega$ such that the trajectory $\left\{\eta_{n}(\omega)\right\}$ goes through $a$ infinitely often. Since $\mathcal{Z}$ is finite we have

$$
\Omega=\bigcup_{a \in \mathcal{Z}} A_{a}^{1} .
$$

If $a$ is visited infinitely often, then according to Theorem 1 , the set $A_{a}^{1}$ can be divided into disjoint subsets $A_{a b}^{2}, b \in \mathcal{E}(a)$, where $A_{a b}^{2}$ is the set of all 
trajectories which go through $a$ and then through $b$ for $n \geq n_{0}(\omega)$. If $b \neq a$, then we can divide $A_{a b}^{2}$ into subsets $A_{a b c}^{3}, c \in \mathcal{E}(b)$, such that for $\omega \in A_{a b c}^{3}$ the trajectory goes successively through $a, b, c$, etc. Since $\mathcal{Z}$ is finite, after some steps the moving point returns to $a$ and we obtain a limit cycle.

Now we give a version of Theorem 2 for infinite graphs. In the infinite case we need some conditions which guarantee that almost all trajectories are bounded.

We assume that $\mathcal{Z}$ is a nonempty countable set and the set $\mathcal{K} \subset \mathcal{Z} \times \mathcal{Z}$ has the following properties:

(a) $(a, b) \in \mathcal{K} \Rightarrow(b, a) \in \mathcal{K}$,

(b) $1 \leq \operatorname{card} \mathcal{E}(a)<\infty$ for $a \in \mathcal{Z}$.

TheOREM 3. Consider a random walk on a graph $(\mathcal{Z}, \mathcal{K})$ which satisfies conditions (a) and (b). Assume that

(i) for each $a \in \mathcal{Z}$ there exists $c_{a}>0$ such that

$$
\bigwedge_{x \in X} \bigvee_{b \in \mathcal{E}(a)} \varphi_{a b}(x) \geq c_{a}
$$

(ii) there exists $c>0$ such that

$$
\bigwedge_{a \in \mathcal{Z}} \bigwedge_{b \in \mathcal{E}(a)} \varphi_{a b}\left(x_{0}\right) \geq c .
$$

Then almost all trajectories have a limit cycle.

Proof. First we give an interpretation of condition (ii). Assume that $A$ is the event that the vertex $a$ is visited for the first at time $t=n_{0}(\omega)$ and the vertex $b \in \mathcal{E}(a)$ has not been visited earlier. Let

$$
B=\left\{\omega \in \Omega: \eta_{n_{0}+2 i-1}(\omega)=b, \eta_{n_{0}+2 i}(\omega)=a \text { for } i=1,2, \ldots\right\} .
$$

Then

$$
\operatorname{Prob}(B \mid A)=\varphi_{a b}\left(x_{0}\right) \varphi_{b a}\left(x_{0}\right) \geq c^{2} .
$$

Now we check that almost all trajectories go through a finite number of vertices. We introduce a distance in the set of vertices. By the length of a chain we mean the number of edges in it. If two vertices $a$ and $b$ are connected by some chain then their distance $d(a, b)$ is the minimal length of a chain connecting $a$ and $b$. If $a$ and $b$ are not connected, then $d(a, b)=\infty$. Assume that the walk begins at a vertex $a_{0}$ and set $m(a)=d\left(a_{0}, a\right)$ for $a \in \mathcal{Z}$. For $k=1,2, \ldots$ we define

$$
C_{k}=\left\{\omega \in \Omega: \sup _{n} m\left(\eta_{n}(\omega)\right) \geq k\right\} .
$$

According to (30) we have

$$
\operatorname{Prob}\left(C_{k+2} \mid C_{k}\right) \leq 1-\varphi_{a b}\left(x_{0}\right) \varphi_{b a}\left(x_{0}\right) \leq 1-c^{2} .
$$


This implies that

$$
\operatorname{Prob}\left(C_{2 k}\right) \leq\left(1-c^{2}\right)^{k}
$$

Let $C=\bigcap_{k=1}^{\infty} C_{k}$. Since $\operatorname{Prob}(C)=0$, we have

$$
\sup _{n} m\left(\eta_{n}(\omega)\right)<\infty \quad \text { for almost all } \omega \in \Omega .
$$

From the assumption (b) it follows that

$$
\operatorname{card}\{a \in \mathcal{Z}: m(a) \leq k\}<\infty \quad \text { for every } k \in \mathbb{N} .
$$

Inequalities (31) and (32) imply that almost every trajectory goes through a finite number of vertices. Similar arguments to those in Theorem 2 show that almost all trajectories have limit cycles.

4. Neural networks. Now we apply our results to a neural network. Let $(\mathcal{Z}, \mathcal{K})$ be a directed graph with a finite set of vertices $\mathcal{Z}$. The graph $(\mathcal{Z}, \mathcal{K})$ is called a neural network if it has the following two properties:

(n1) if $\left(a_{1}, \ldots, a_{k}\right), k \geq 2$, is a sequence of vertices such that $\left(a_{i}, a_{i+1}\right) \in$ $\mathcal{K}$ for $i=1, \ldots, k-1$, then $a_{1} \neq a_{k}$,

(n2) for each $a \in \mathcal{Z}$ there exists $b \in \mathcal{Z}$ such that $(a, b) \in \mathcal{K}$ or $(b, a) \in \mathcal{K}$.

Now we divide the set of vertices into three disjoint subsets:

$$
\begin{aligned}
& \mathcal{Z}_{b}=\{a \in \mathcal{Z}: \mathcal{B}(a)=\emptyset\}, \quad \mathcal{Z}_{e}=\{a \in \mathcal{Z}: \mathcal{E}(a)=\emptyset\}, \\
& \mathcal{Z}_{i}=\mathcal{Z} \backslash\left(\mathcal{Z}_{b} \cup \mathcal{Z}_{e}\right) .
\end{aligned}
$$

From (n1) it follows that $\mathcal{Z}_{b} \neq \emptyset$ and $\mathcal{Z}_{e} \neq \emptyset$. Elements of $\mathcal{Z}_{b}$ are called input vertices and elements of $\mathcal{Z}_{e}$ are called output vertices. Fig. 1 shows an example of a neural network.

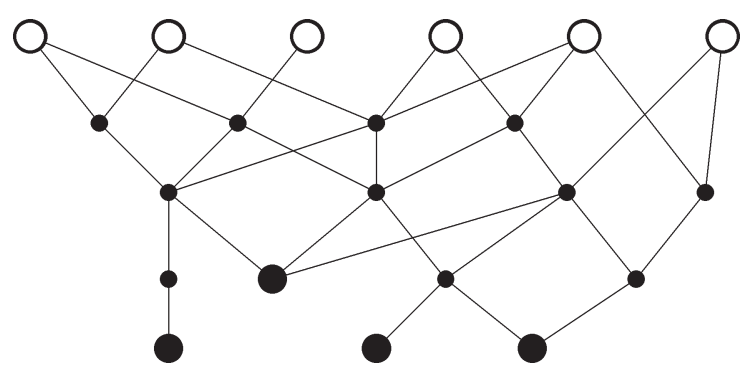

Fig. 1. $\bigcirc$ : input vertices $\mathcal{Z}_{b}, \bullet$ : output vertices $\mathcal{Z}_{e}, \bullet$ : vertices from the set $\mathcal{Z}_{i}$

The neutral network $(\mathcal{Z}, \mathcal{K})$ is taught in the following way. For each vertex $a \in \mathcal{Z}_{b} \cup \mathcal{Z}_{i}$ we introduce a random decision system

$$
\mathcal{S}_{a}=\left(X, \mathcal{A},\left\{P_{a b}\right\}_{b \in \mathcal{E}(a)},\left\{p_{a b}\right\}_{b \in \mathcal{E}(a)}\right) .
$$

Let $\mathcal{Z}_{b}=\left\{b^{1}, \ldots, b^{r}\right\}$. We choose an element $b^{i}$ with a probability $q_{i}$, where $q_{i}>0$ and $q_{1}+\cdots+q_{r}=1$. Then we go through the graph $(\mathcal{Z}, \mathcal{K})$ according 
to the procedure described in Section 3 using the random decision systems $\mathcal{S}_{a}, a \in \mathcal{Z}_{b} \cup \mathcal{Z}_{i}$, unless we reach a vertex in $\mathcal{Z}_{e}$. Then we choose an element $b^{i}$ with a probability $q_{i}$ and we go through the graph $(\mathcal{Z}, \mathcal{K})$ etc. As in Section 3 , we denote by $\eta_{n}$ the position of the moving point at time $t=n$.

A neural network $(\mathcal{Z}, \mathcal{K})$ is called simple if for each $a \in \mathcal{Z}_{b} \cup \mathcal{Z}_{i}$ there exists exactly one $b \in \mathcal{Z}_{e} \cup \mathcal{Z}_{i}$ such that $(a, b) \in \mathcal{K}$. Observe that if a neural network is simple then the movement on it is strictly determined. A neural network $\left(\mathcal{Z}^{\prime}, \mathcal{K}^{\prime}\right)$ is called a subnetwork of $(\mathcal{Z}, \mathcal{K})$ if $\mathcal{Z}^{\prime} \subset \mathcal{Z}, \mathcal{K}^{\prime} \subset \mathcal{K}$ and $\mathcal{Z}_{b}^{\prime}=\mathcal{Z}_{b}$. Thick line segments on Fig. 2 form a simple subnetwork of the neural network from Fig. 1.

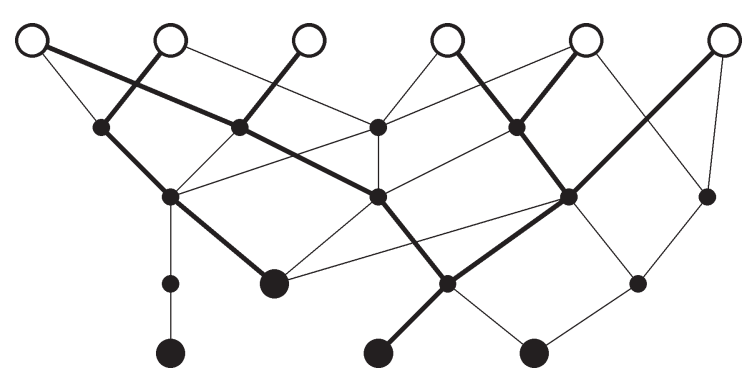

Fig. 2

TheORem 4. Consider a neural network $(\mathcal{Z}, \mathcal{K})$ and let $c>0$ be a given number. Assume that for each $a \in \mathcal{Z}_{b} \cup \mathcal{Z}_{i}$ and $x \in X$ there is $b \in \mathcal{E}(a)$ such that $\varphi_{a b}(x) \geq c$. Then there exists a measurable subset $\Omega_{0} \subset \Omega$ such that $\operatorname{Prob}\left(\Omega_{0}\right)=1$ and for each $\omega \in \Omega_{0}$ there exist an $n_{0} \in \mathbb{N}$ and a simple subnetwork $\left(\mathcal{Z}^{\prime}, \mathcal{K}^{\prime}\right)$ of $(\mathcal{Z}, \mathcal{K})$ such that $\eta_{n} \in \mathcal{Z}^{\prime}$ for $n \geq n_{0}$.

Proof. The proof is the same as that of Theorem 2.

\section{References}

[1] M. Benaïm, Vertex-reinforced random walks and conjecture of Pemantle, Ann. Probab. 25 (1997), 361-392.

[2] M. Cranston and Y. Le Jan, Self-attracting diffusions: Two case studies, Math. Ann. 303 (1995), 87-93.

[3] D. Coppersmith and P. Diaconis, Random walks with reinforcement, unpublished manuscript, 1987.

[4] B. Davis, Reinforced random walk, Probab. Theory Related Fields 84 (1990), 203229.

[5] B. Davis and S. Volkov, Vertex-reinforced jump processes on trees and finite graphs, Probab. Theory Related Fields 128 (2004), 42-62.

[6] D. O. Hebb, The Organization of Behavior, Wiley, New York, 1949.

[7] F. C. Hoppensteadt and E. M. Izhikevich, Weakly Connected Neural Networks, Appl. Math. Sci. 126, Springer, New York, 1997. 
[8] L. Horváth and Q.-M. Shao, Limit distributions of directionally reinforced random walks, Adv. Math. 134 (1998), 367-383.

[9] M. Iosifescu and R. Theodorescu, Random Processes and Learning, Springer, New York, 1969.

[10] M. S. Keane and S. W. W. Rolles, Edge-reinforced random walk on finite graphs, in: Infinite Dimensional Stochastic Analysis, Ph. Clément et al. (eds.), Koninklijke Nederlandse Akad. Wetensch. 52, 217-234.

[11] —, 一, Tubular recurrence, Acta Math. Hungar. 97 (2002), 207-221.

[12] V. Limic, Attracting edge property for a class of reinforced random walks, Ann. Probab. 31 (2003), 1615-1654.

[13] R. Lyons and R. Pemantle, Random walk in a random environment and first-passage percolation on trees, ibid. 20 (1992), 125-136.

[14] N. Madras and G. Slade, The Self-Avoiding Walk, Birkhäuser, Boston, 1993.

[15] R. D. Mauldin, M. Monticino, and H. von Weizsäcker, Directionally reinforced random walks, Adv. Math. 117 (1996), 239-252.

[16] R. Pemantle, Phase transition in reinforced random walk and RWRE on trees, Ann. Probab. 16 (1988), 1229-1241.

[17] —, Vertex reinforced random walk, Probab. Theory Related Fields 92 (1992), 117136.

[18] O. Raimond, Self-attracting diffusions: Case of the constant interaction, ibid. 107 (1997), 177-196.

[19] P. Révész and B. Tóth (eds.), Random Walks (Budapest, 1998), Bolyai Soc. Math. Stud. 9, János Bolyai Math. Soc., Budapest, 1999.

[20] R. Rudnicki and M. Wolf, Random walk with memory, J. Math. Phys. 40 (1999), 3072-3083.

[21] T. Sellke, Reinforced random walk on d-dimensional integer lattice, Tech. Rept. No. 94-26, Dept. of Statistics, Purdue Univ.

[22] B. Tóth, Self-interacting random motions, in: Eur. Congr. Math., Vol. I (Barcelona, 2000), Progr. Math. 201, Birkhäuser, Basel, 2001, 555-564.

[23] S. Volkov, Vertex-reinforced random walk on arbitrary graphs, Ann. Probab. 29 (2001), 66-91.

Dipartimento di Matematica Pura ed Applicata

Università di L'Aquila

Via Vetoio, 67100 L'Aquila, Italy

E-mail: myjak@univaq.it

and

WMS AGH

al. Mickiewicza 30

30-059 Kraków, Poland
Institute of Mathematics

Polish Academy of Sciences

Bankowa 14

40-007 Katowice, Poland and

Institute of Mathematics Silesian University

Bankowa 14

40-007 Katowice, Poland

E-mail: rudnicki@us.edu.pl 